\title{
Separability of a mixture of Dicke states
}

\author{
Nengkun $\mathrm{Yu}^{*}$ \\ Centre for Quantum Software and Information, Faculty of Engineering and Information Technology, University of Technology Sydney, 15 \\ Broadway, Ultimo, New South Wales 2007, Australia; \\ Institute for Quantum Computing, University of Waterloo, 200 University Avenue W, Waterloo, Ontario N2L 3G1, Canada; \\ and Department of Mathematics \& Statistics, University of Guelph, 50 Stone Road E, Guelph, Ontario N1G 2W1, Canada
}

(Received 14 March 2016; published 16 December 2016)

\begin{abstract}
The structural relation between multipartite entanglement and symmetry is one of the central mysteries of quantum mechanics. In this paper, we study the separability of quantum states in the bosonic system. We show that a mixture of multiqubit Dicke states is separable if and only if its partial transpose is positive semidefinite, which confirms the hypothesis of Wolfe and Yelin [E. Wolfe and S. F. Yelin, Phys. Rev. Lett. 112, 140402 (2014)]. We generalize this result to a class of bosonic states in the $d \otimes d$ system; and for general $d$, we determine its separability is NP-hard although verifiable conditions for separability are easily derived when $d=3,4$.
\end{abstract}

DOI: 10.1103/PhysRevA.94.060101

Introduction. Quantum entanglement has been regarded as a resource of cryptography and metrology [1,2]. Therefore, it is a fundamental problem to qualitatively test whether a given state is entangled or not. In multipartite systems, a quantum state is called fully separable, not entangled, if it can be written as a statistical mixture of product states. Although it is known to be NP-hard of testing separability [3], a considerable number of different separability criteria have been discovered (see the references in [4,5]), including the famous positive partial transpose (PPT) criterion [6]. One widely used tool of detecting entanglement is the entanglement witness [7,8]. Another key concept for entanglement detection is symmetry. The $k$-symmetric extension provides a hierarchy of separability criteria [9-13], which converges exactly to the set of separable states when $k$ goes to infinity.

Due to the essential role of symmetry played in entanglement theory, it becomes of great interest to study the relation between multipartite entanglement and symmetry, more precisely, the entanglement of bosonic systems. For $N$-qubit bosonic systems, a natural basis is the $N$-qubit Dicke state (unnormalized),

$$
\left|D_{N, n}\right\rangle:=\left(\begin{array}{c}
N \\
n
\end{array}\right) P_{\text {sym }}\left(|0\rangle^{\otimes n} \otimes|1\rangle^{\otimes N-n}\right),
$$

with $P_{\text {sym }}$ being the projection onto the bosonic (fully symmetric) subspace, i.e., $P_{\text {sym }}=\frac{1}{N !} \sum_{\pi \in S_{N}} U_{\pi}$, the sum extending over all permutation operators $U_{\pi}$ of the $N$-qubit systems. Dicke states are particularly suitable for cold atomic systems, where the particle number is usually thousands. Considerable effort has been devoted to the study of entanglement of Dicke states, both theoretically [14-20] and experimentally [21-26]. The separability of bosonic states, especially the role of PPT in the separability of bosonic systems, has attracted a lot of attention. Eckert et al. proved that there is no PPT entanglement in three-qubit bosonic system [14]. PPT entanglement was found in five- and six-qubit bosonic systems in [27]. The existence of four-qubit bosonic PPT entanglement is demonstrated in Ref. [28]. Particularly, analytical criteria

\footnotetext{
*nengkunyu@gmail.com
}

of the separability of the mixture of Dicke states have been pursued extensively [27,29-32]. For instance, in Ref. [30], Quesada et al. provided the analytical expression for the best separable approximation of a mixture of Dicke states by using the idea introduced by Lewenstein et al. in [31]. In Ref. [32], Wolfe and Yelin proposed the hypothesis that a mixture of Dicke states is separable if and only if it is PPT, according to their ideas on generating sufficient separability criteria numerically.

In this paper, we confirm the validity of the hypothesis that PPT indicates separability of a mixture of Dicke states by giving a two-step proof. At the first step, we show that a mixture of Dicke states is separable if and only if two Hankel matrices [33] generated by its eigenvalues is positive semidefinite. Second, by using the relation between Dicke states, we demonstrate that PPT of a mixture of Dicke states implies the positive semidefinite of the two Hankel matrices. These two parts directly lead us to the conclusion that PPT is a sufficient and necessary condition of the separability of a mixture of Dicke states. Notably, in order to ensure the positive semidefinite of the two Hankel matrices, we only need to take the partial transpose in the half cut of the subsystems. Our main tool in the proof of the first step is to give a complete characterization of the general entanglement witness of the class of Dicke states by studying the non-negative polynomials. This idea is generalized to prove that the separability of a mixture of bipartite high-dimensional Dicke states is NPcomplete, although a very simple criterion is given when the local dimension is three or four.

Main results. In the $N$-qudit system $\mathcal{H}_{1} \otimes \mathcal{H}_{2} \otimes \cdots \otimes \mathcal{H}_{N}$ with $d$ being the dimension of each Hilbert space $\mathcal{H}_{i}$, the bosonic space is a subspace of pure states which are invariant under the swap of any two subsystems among all $N$ subsystems, i.e., for a swap operator $F_{i, j}$ exchanging a two-qudit system,

$$
S: \equiv\left\{|\psi\rangle:|\psi\rangle=F_{i, j}|\psi\rangle, \text { for all } i, j\right\} .
$$

In the $d=2$ case, the symmetric space is spanned by Dicke states $\left|D_{N, 0}\right\rangle, \ldots,\left|D_{N, N}\right\rangle$. A mixed state $\rho$ is called bosonic if its support is a subspace of bosonic space where the support of $\rho$ is the subspace spanned by the eigenvectors corresponding 
to its nonzero eigenvalues. In other words, $\rho=F_{i, j} \rho=\rho F_{i, j}$ holds for $1 \leqslant i, j \leqslant N$.

An $N$-qubit bosonic state $\rho$ is called fully separable, if $\rho$ can be written as $\rho=\sum_{j} p_{j} \otimes_{k=1}^{N}\left|\alpha_{j_{k}}\right\rangle\left\langle\alpha_{j_{k}}\right|$,

$$
\otimes_{k=1}^{N}\left|\alpha_{j_{k}}\right\rangle \in S \Rightarrow \exists\left|\alpha_{j}\right\rangle, \quad \otimes_{k=1}^{N}\left|\alpha_{j_{k}}\right\rangle=\left|\alpha_{j}\right\rangle^{\otimes N} .
$$

That is, $\rho=\sum_{j} p_{j} \alpha_{j}^{\otimes N}$ with $\alpha_{j}=\left|\alpha_{j}\right\rangle\left\langle\alpha_{j}\right|$.

Now, we study general entanglement witnesses for a bosonic system as a useful tool which was introduced in [27]. For the $N$-qudit system, a Hermitian $W$ is called a general entanglement witness of the bosonic system if $W=P_{S} W P_{S}$ with $P_{S}$ being the projection onto the bosonic space $S$ and

$$
\operatorname{tr}\left(W \alpha^{\otimes N}\right) \geqslant 0, \quad \text { for all } \alpha=|\alpha\rangle\langle\alpha| .
$$

By invoking the hyperplane separation theorem, we make the followng proposition:

Proposition 1. A bosonic state $\rho$ is separable if and only if $\operatorname{tr}(W \rho) \geqslant 0$ holds for any general entanglement witness $W$ of the bosonic system.

Proof. The "only if" part follows directly. For the "if" part, assume there is some bosonic entangled state $\rho$ such that $\operatorname{tr}(W \rho) \geqslant 0$ holds for any general entanglement witness $W$. Separable states of the bosonic system is convex and compact. By the hyperplane separation theorem, there exists $H$ such that $\operatorname{tr}(H \rho)<0$ and $\operatorname{tr}\left(H \alpha^{\otimes N}\right) \geqslant 0$ holds for any $\alpha$. Then $W=P_{S} H P_{S}$ is a general entanglement witness. On the other hand, $\operatorname{tr}(W \rho)=\operatorname{tr}\left(P_{S} H P_{S} \rho\right)=\operatorname{tr}(H \rho)<0$, which contradicts the assumption.

In the following, we mainly focus on the $N$-qubit bosonic system, that is, $d=2$. Naturally, the set of general entanglement witnesses forms a convex cone, i.e., a positive combination of two general entanglement witnesses is still a general entanglement witness.

One way to test the separability of bosonic states is to parametrize the set of general entanglement witnesses, at least its boundary. Notice that any Hermitian $W=P_{S} W P_{S}$ corresponds to a Hermitian matrix $M:=\left(m_{i, j}\right)_{(N+1) \times(N+1)}$ as follows:

$$
W:=\sum_{i, j=0}^{N} m_{i, j}\left|\widetilde{D_{N, i}}\right\rangle\left\langle\widetilde{D_{N, j}}\right|
$$

where $\left|\widetilde{D_{N, n}}\right\rangle:=\left(\begin{array}{c}N \\ n\end{array}\right)^{-1}\left|D_{N, n}\right\rangle$, i.e., $\left\langle D_{N, m} \mid \widetilde{D_{N, n}}\right\rangle=\delta_{m, n}$, the Dirac delta function.

The condition of $W$ being a general entanglement witness $\operatorname{tr}\left(W \alpha^{\otimes N}\right) \geqslant 0$ holds for all one-qubit $|\alpha\rangle$. That is, for any $z \in \mathbb{C}$,

$$
\begin{gathered}
\operatorname{tr}\left(W|0\rangle\left\langle\left. 0\right|^{\otimes N}\right) \geqslant 0 \Leftrightarrow m_{N, N} \geqslant 0,\right. \\
\operatorname{tr}\left\{W\left[(|1\rangle+z|0\rangle)\left(\langle 1|+z^{*}\langle 0|\right)\right]^{\otimes N}\right\} \geqslant 0 \\
\Leftrightarrow \vec{z}^{\dagger} M \vec{z} \geqslant 0 \quad \text { with } \vec{z}=\left(1, z, z^{2}, \ldots, z^{N}\right)^{T} \in \mathbb{C}^{N+1} .
\end{gathered}
$$

The second condition indicates the first as $|z| \rightarrow \infty$. The last equivalence condition is derived from the fact that $(|1\rangle+z|0\rangle)^{\otimes N}=\sum_{j=0}^{N} z^{j}\left|D_{N, j}\right\rangle$.

By employing this condition, we provide a necessary and sufficient analytical condition for $N$-qubit separability of the mixture of Dicke states, which was called diagonal symmetric states in previous literature [27-30,32],

$$
\rho=\sum_{n=0}^{N} \chi_{n}\left|D_{N, n}\right\rangle\left\langle D_{N, n}\right|
$$

Such $\rho$ enjoys the property that for all diagonal qubit unitary $U_{\theta}=\operatorname{diag}\left\{1, e^{i \theta}\right\}$,

$$
\rho=U_{\theta}^{\otimes N} \rho U_{\theta}^{\dagger \otimes N}
$$

Thus, for any general entanglement witness $W$, we construct a "diagonal" general entanglement witness $W_{0}$ with $\operatorname{tr}\left(W_{0} \rho\right)=$ $\operatorname{tr}(W \rho)$ by

$$
W_{0}=\frac{1}{2 \pi} \int_{0}^{2 \pi} U_{\theta}^{\dagger \otimes N} W U_{\theta}^{\otimes N} d \theta=\sum_{k=0}^{N} m_{k, k}\left|\widetilde{D_{N, k}}\right\rangle\left\langle\widetilde{D_{N, k} \mid} .\right.
$$

$\operatorname{tr}(W \rho)=\operatorname{tr}\left(W U_{\theta}^{\otimes N} \rho U_{\theta}^{\dagger \otimes N}\right)=\operatorname{tr}\left(U_{\theta}^{\dagger \otimes N} W U_{\theta}^{\otimes N} \rho\right)=\operatorname{tr}\left(W_{0} \rho\right)$.

Proposition 1 indicates the following:

Proposition 2. The $N$-qubit mixture of Dicke states $\rho$ is separable if and only if $\operatorname{tr}\left(W_{0} \rho\right) \geqslant 0$ for any diagonal general entanglement witness $W_{0}$.

For any $W_{0}=\sum_{k=0}^{N} m_{k, k}\left|\widetilde{D_{N, k}}\right\rangle\left\langle\widetilde{D_{N, k}}\right|$, we define its corresponding real coefficient polynomial

$$
g(x):=\sum_{k=0}^{N} m_{k, k} x^{k}
$$

Then, invoking Eq. (1), $W_{0}$ is a diagonal general entanglement witness if and only if $\sum_{k=0}^{N} m_{k, k}|z|^{2 k}$ is always non-negative for all $z \in \mathbb{C}$. That is,

$$
g(r) \geqslant 0 \forall r \geqslant 0 .
$$

The characterization of such polynomials is accomplished by the following proposition:

Proposition 3. A real coefficient polynomial $g(x)$ satisfies that $g(r) \geqslant 0$ for all $r \geqslant 0$, if and only if there exists a real coefficient polynomial $P_{i}(x), Q_{i}(x)$ such that

$$
g(x)=\sum_{i} x P_{i}^{2}(x)+\sum_{i} Q_{i}^{2}(x) .
$$

Proof. The "if" part is direct. For the "only if" part, we use the fundamental theorem of algebra,

$$
g(x)=a_{0} \prod\left(x-z_{k}\right)^{l_{k}} .
$$

For non-real root $z_{k}$, we know that for all real $r$,

$$
\left(r-z_{k}\right)\left(r-\overline{z_{k}}\right)=\left[r-\operatorname{Re}\left(z_{k}\right)\right]^{2}+\operatorname{Im}^{2}\left(z_{k}\right) \geqslant 0 .
$$

For nonpositive $z_{k}$, we know that for all $r \geqslant 0$,

$$
r-z_{k}=r+\left(-z_{k}\right) \geqslant 0 .
$$

For positive $z_{k}$, its power $l_{k}$ must be even according to $g(r) \geqslant$ 0 for all $r \geqslant 0$. Then, expanding $g(x)=a_{0} \prod\left(x-z_{k}\right)^{l_{k}}$, we know that $g(x)$ has the wanted form.

Invoking the relation between the diagonal general entanglement witness $W_{0}$ and $g(x)$, one can deduce the following:

Proposition 4. Any diagonal general entanglement witness for an $N$-qubit mixture of Dicke states can be written as a 
convex combination of the following two types of general entanglement witnesses:

$$
\begin{aligned}
R & =\sum_{0 \leqslant i, j \leqslant \frac{N}{2}} a_{i} a_{j}\left|\widetilde{D_{N, i+j}}\right\rangle\left\langle\widehat{D_{N, i+j}}\right|, \\
T & =\sum_{0 \leqslant i, j \leqslant \frac{N-1}{2}} b_{i} b_{j}\left|\widetilde{D_{N, i+j+1}}\right\rangle\left\langle\widetilde{D_{N, i+j+1}}\right|,
\end{aligned}
$$

with $a_{k}, b_{k} \in \mathbb{R}$. Here we correspond $R$ and $T$ to $Q(x)^{2}$ and $x P(x)^{2}$, respectively, with $Q(x)=\sum_{k=0}^{\frac{N}{2}} a_{i} x^{i}$ and $P(x)=$ $\sum_{k=0}^{\frac{N-1}{2}} b_{i} x^{i}$. Now we are ready to show our main result:

Theorem 1. The mixture of Dicke states $\rho=$ $\sum_{n=0}^{N} \chi_{n}\left|D_{N, n}\right\rangle\left\langle D_{N, n}\right|$ is separable if and only if the following two Hankel matrices [33] $M_{0}, M_{1}$ are positive semidefinite, i.e.,

$$
\begin{aligned}
M_{0} & :=\left(\begin{array}{ccc}
\chi_{0} & \cdots & \chi_{m_{0}} \\
\cdots & \cdots & \cdots \\
\chi_{m_{0}} & \cdots & \chi_{2 m_{0}}
\end{array}\right) \geqslant 0, \\
M_{1} & :=\left(\begin{array}{ccc}
\chi_{1} & \cdots & \chi_{m_{1}} \\
\cdots & \cdots & \cdots \\
\chi_{m_{1}} & \cdots & \chi_{2 m_{1}-1}
\end{array}\right) \geqslant 0,
\end{aligned}
$$

where $m_{0}:=\left[\frac{N}{2}\right]$ and $m_{1}:=\left[\frac{N+1}{2}\right]$.

Proof. According to Propositions 2 and 4, $\rho$ is separable if and only if $\operatorname{tr}\left(W_{0} \rho\right) \geqslant 0$ holds for any extreme point of diagonal general entanglement witness, that is, $R$ and $T$ types of general entanglement witnesses. Equivalently, for all $\vec{a}=\left(a_{0}, \ldots, a_{m_{0}}\right)^{T} \in \mathbb{R}^{m_{0}+1}$ and $\vec{b}=\left(b_{1}, \ldots, b_{m_{1}}\right)^{T} \in \mathbb{R}^{m_{1}}$ the following quadratic forms are non-negative:

$$
\begin{aligned}
& \operatorname{tr}(R \rho)=\sum_{0 \leqslant i, j \leqslant m_{0}} \chi_{i+j} a_{i} a_{j}=\vec{a}^{T} M_{0} \vec{a} \geqslant 0, \\
& \operatorname{tr}(T \rho)=\sum_{1 \leqslant i, j \leqslant m_{1}} \chi_{i+j-1} b_{i} b_{j}=\vec{b}^{T} M_{1} \vec{b} \geqslant 0 .
\end{aligned}
$$

Notice that $M_{0}, M_{1}$ are real matrices. The above condition is equivalent to $M_{0}, M_{1} \geqslant 0$.

In addition, we know the following:

Theorem 2. An $N$-qubit mixture of Dicke states $\rho=$ $\sum_{n=0}^{N} \chi_{n}\left|D_{N, n}\right\rangle\left\langle D_{N, n}\right|$ is separable if and only if it is PPT. More precisely, $\rho$ is separable if and only if it is PPT under the partial transpose of $m_{0}=\left[\frac{N}{2}\right]$ subsystems.

Remark: A positive semidefinite matrix $M$ acting on a bipartite system is called PPT if $M^{\Gamma} \geqslant 0$ holds, where $\Gamma$ means the partial transpose, i.e., $(|i j\rangle\langle k l|)^{\Gamma_{\mathcal{A}}}=|k j\rangle\langle i l|$.

Proof. Assume $\rho$ is positive under the partial transpose of the first $m_{0}=\left[\frac{N}{2}\right]$ subsystems, according to Theorem 1, we only need to show $M_{0}, M_{1} \geqslant 0$ of Eqs. (1) and (2).

Write $\rho^{\Gamma}$ in basis $\left|D_{m_{0}, j}\right\rangle\left|D_{m_{1}, k}\right\rangle$ by verifying the following relation between Dicke states:

$$
\left|D_{N, n}\right\rangle=\sum_{j=\max \left\{0, n-m_{1}\right\}}^{\min \left\{n, m_{0}\right\}}\left|D_{m_{0}, j}\right\rangle\left|D_{m_{1}, n-j}\right\rangle .
$$

Since $\rho^{\Gamma} \geqslant 0$, then the restriction of $\rho^{\Gamma}$ on the subspace spanned by $\left\{\left|D_{m_{0}, j}\right\rangle\left|D_{m_{1}, j}\right\rangle, 0 \leqslant j \leqslant m_{0}\right\}$ is non-negative. Direct calculation indicates $M_{0} \geqslant 0$.

On the other hand, the restriction of $\rho^{\Gamma}$ on the subspace spanned by $\left\{\left|D_{m_{0}, j-1}\right\rangle\left|D_{m_{1}, j}\right\rangle, 1 \leqslant j \leqslant m_{1}\right\}$ is non-negative. That leads us to $M_{1} \geqslant 0$. Therefore, $\rho^{\Gamma}$ implies the separability of $\rho$.
If bosonic state $\rho$ is separable, then the mixture of Dicke states $\sigma=\int U^{\otimes N} \rho U^{\dagger \otimes N} d U$ is separable, where $d U$ ranges over the diagonal qubit unitaries. Then, Theorem 2 indeed provides a necessary condition on the separability of the general $N$-qubit bosonic state.

These techniques to study multiqubit Dicke states can be generalized to study the mixture of higher dimensional bipartite Dicke states,

$$
\rho=\sum_{i, j=1}^{d} \chi_{i, j}\left|\psi_{i, j}\right\rangle\left\langle\psi_{i, j}\right|,
$$

with $\left|\psi_{i, j}\right\rangle:=\left\{\begin{array}{ll}|i i\rangle & \text { if } i=j, \\ |i j\rangle+|j i\rangle & \text { otherwise. }\end{array}\right.$ being some basis of the $d \otimes d$ symmetric subspace.

By using that $\rho=(U \otimes U) \rho(U \otimes U)^{\dagger}$ holds for all diagonal qudit unitary $U$, we know the following:

$$
\begin{aligned}
\rho & =\sum_{k} \alpha_{k}^{\otimes 2} \\
& =\sum_{k} \int(U \otimes U) \alpha_{k}^{\otimes 2}(U \otimes U)^{\dagger} d U \\
& =\sum_{k}\left|x_{k, i}\right|^{2}\left|x_{k, j}\right|^{2}\left|\psi_{i, j}\right\rangle\left\langle\psi_{i, j}\right|, \\
\Leftrightarrow \chi: & =\left(\chi_{i j}\right)_{d \times d}=\sum_{k} \vec{x}_{k} \vec{x}_{k}^{T},
\end{aligned}
$$

where $d U$ ranges over all diagonal unitaries, $\left|\alpha_{k}\right\rangle=\sum_{j=1}^{d} x_{k, j}|j\rangle, \alpha_{k}=\left|\alpha_{k}\right\rangle\left\langle\alpha_{k}\right|, \quad$ and $\quad \vec{x}_{k}=$ $\left(\left|x_{k, 1}\right|^{2}, \ldots,\left|x_{k, d}\right|^{2}\right)^{T} \in \mathbb{R}_{+}^{d}$, with $\mathbb{R}_{+}^{d}$ standing for the $d$-dimensional vector space whose entries are non-negative.

The above argument indicates that $\rho$ is separable if and only if $\chi$ is a completely positive matrix, where the cone of completely positive matrices [34] is defined as

$$
\mathcal{C}=\left\{\sum_{i} \vec{y}_{k} \vec{y}_{k}^{T}: \vec{y}_{k} \in \mathbb{R}_{+}^{d}\right\} .
$$

Recalling the known hardness result on testing the membership of completely positive matrices in Refs. $[35,36]$, we have the following:

Theorem 3. It is NP-hard to decide whether $\rho=$ $\sum_{i, j=1}^{d} \chi_{i, j}\left|\psi_{i, j}\right\rangle\left\langle\psi_{i, j}\right|$ is separable. On the other hand, for $d=$ 3,4 , it is separable if and only if $\chi=\left(\chi_{i j}\right)_{d \times d}$ is semi-definite positive.

Conclusion. In this paper, we study the separability of bosonic state. We prove the validity of the hypothesis of Ref. [32] by demonstrating an analytical condition for the separability of a mixture of $N$-qubit Dicke states. These techniques are also applied to the mixture of $d \otimes d$ Dicke states, and the hardness result is shown. We hope that our techniques for certifying entanglement witnesses and positive polynomials may prove useful in furthering the understanding of entanglement. One interesting goal is to provide complete criteria for the separability of general $N$-qubit bosonic states.

Acknowledgments. We thank John Watrous, Debbie Leung, Bei Zeng, and Youming Qiao for their helpful discussions. N.Y. is supported by the Natural Sciences and Engineering Research Council of Canada, Natural Sciences and Engineering Research Council Discovery Accelerator, Canada Research Chairs, and the Canadian Institute for Advanced Research. 
[1] C. H. Bennett and G. Brassard, in International Conference on Computers, Systems, \& Signal Processing: Proceedings, Dec. 9-12, 1984, Bangalore, India (IEEE, New York, 1984).

[2] C. Gross, T. Zibold, E. Nicklas, J. Esteve, and M. K. Oberthaler, Nature 464, 1165 (2010).

[3] L. Gurvits, J. Comput. Syst. Sci. 69, 448 (2004).

[4] L. M. Ioannou, Quantum Inf. Comput. 7, 335 (2007).

[5] R. Horodecki, P. Horodecki, M. Horodecki, and K. Horodecki, Rev. Mod. Phys. 81, 865 (2009).

[6] A. Peres, Phys. Rev. Lett. 77, 1413 (1996).

[7] M. Horodecki, P. Horodecki, and R. Horodecki, Phys. Lett. A 223, 1 (1996); 283, 1 (2001).

[8] B. M. Terhal, Phys. Lett. A 271, 319 (2000).

[9] A. C. Doherty, P. A. Parrilo, and F. M. Spedalieri, Phys. Rev. Lett. 88, 187904 (2002).

[10] M. Christandl, R. Konig, G. Mitchison, and R. Renner, Commun. Math. Phys. 273, 473 (2007).

[11] G. Chiribella, in Lecture Notes in Computer Science, Vol. 6519 (Springer, New York, 2011), p. 9.

[12] A. W. Harrow, arXiv:1308.6595.

[13] F. G. S. L. Brãndao, and M. Christandl, Phys. Rev. Lett. 109, 160502 (2012)

[14] K. Eckert, J. Schliemann, D. Bruss, and M. Lewenstein, Ann. Phys. (NY) 299, 88 (2002).

[15] N. Yu, Phys. Rev. A 87, 052310 (2013); N. Yu, E. Chitambar, C. Guo, and R. Duan, ibid. 81, 014301 (2010); N. Yu, C. Guo, and R. Duan, Phys. Rev. Lett 112, 160401 (2014).

[16] W. Dür, G. Vidal, and J. I. Cirac, Phys. Rev. A 62, 062314 (2000).

[17] R. Hübener, M. Kleinmann, T.-C. Wei, C. GonzálezGuillén, and O. Gühne, Phys. Rev. A 80, 032324 (2009).

[18] T. Bastin, S. Krins, P. Mathonet, M. Godefroid, L. Lamata, and E. Solano, Phys. Rev. Lett. 103, 070503 (2009); T. Bastin, C. Thiel, J. von Zanthier, L. Lamata, E. Solano, and G. S. Agarwal, ibid. 102, 053601 (2009).
[19] W. Wieczorek, N. Kiesel, C. Schmid, and H. Weinfurter, Phys. Rev. A 79, 022311 (2009).

[20] J. K. Stockton, J. M. Geremia, A. C. Doherty, and H. Mabuchi, Phys. Rev. A 67, 022112 (2003).

[21] M. Cramer, A. Bernard, N. Fabbri, L. Fallani, C. Fort, S. Rosi, F. Caruso, M. Inguscio, and M. B. Plenio, Nat. Commun. 4, 2161 (2013).

[22] R. McConnell, H. Zhang, J. Hu, S. Ćuk, and V. Vuletić, Nature 519, 439 (2015).

[23] C. Lu, Xiao-Qi Zhou, O. Gühne, W. Gao, J. Zhang, Z. Yuan, A. Goebel, T. Yang, and J. Pan, Nat. Phys. 3, 91 (2007).

[24] W. Wieczorek, R. Krischek, N. Kiesel, P. Michelberger, G. Tóth, and H. Weinfurter, Phys. Rev. Lett. 103, 020504 (2009).

[25] R. Prevedel, G. Cronenberg, M. S. Tame, M. Paternostro, P. Walther, M. S. Kim, and A. Zeilinger, Phys. Rev. Lett. 103, 020503 (2009).

[26] C. D. Hamley, C. S. Gerving, T. M. Hoang, E. M. Bookjans, and M. S. Chapman, Nat. Phys. 8, 305 (2012).

[27] G. Tóth and O. Gühne, Appl. Phys. B 98, 617 (2009); Phys. Rev. Lett. 102, 170503 (2009); O. Gühne and G. Tóth, Phys. Rep. 474, 1 (2009).

[28] J. Tura, R. Augusiak, P. Hyllus, M. Kus, J. Samsonowicz, and M. Lewenstein, Phys. Rev. A 85, 060302(R) (2012).

[29] L. Novo, and T. Moroder, and O. Gühne, Phys. Rev. A 88, 012305 (2013).

[30] R. Quesada and A. Sanpera, Phys. Rev. A 89, 052319 (2014).

[31] M. Lewenstein and A. Sanpera, Phys. Rev. Lett. 80, 2261 (1998).

[32] E. Wolfe and S. F. Yelin, Phys. Rev. Lett. 112, 140402 (2014); E. Wolfe, arXiv:1409.2517; E. Wolfe and S. F. Yelin, arXiv:1405.5288.

[33] J. R. Partington, An Introduction to Hankel Operators, London Mathematical Society Student Texts 13 (Cambridge University, New York, 1988).

[34] L. J. Gray and D. G. Wilson, Lin. Algebra Appl. 31, 119 (1980).

[35] P. J. C. Dickinson, and L. Gijben, Comput. Optim. Appl. 57, 403 (2014).

[36] H. Diananda, Math. Proc. Camb. Phil. Soc. 58, 17 (1961). 